\title{
Ipilimumab and Pembrolizumab Mixed Response in a 41-Year-Old Patient with SMARCA4-Deficient Thoracic Sarcoma: An Interdisciplinary Case Study
}

\author{
Nina Anžičc ${ }^{a}$ Fatime Krasniqi ${ }^{b}$ Anna-Lena Eberhardt ${ }^{c}$ \\ Alexandar Tzankov ${ }^{a}$ Jasmin Dionne Haslbauer ${ }^{a}$ \\ aInstitute of Pathology, University Hospital Basel, Basel, Switzerland; ${ }^{b}$ Centre of Oncology, \\ University Hospital Basel, Basel, Switzerland; ' ${ }^{\circ}$ entre of Radio-Oncology, University Hospital \\ Basel, Basel, Switzerland
}

\section{Keywords}

SMARCA4 · SMARCA4-deficient thoracic sarcoma · Pembrolizumab · Ipilimumab ·

Checkpoint inhibitor

\begin{abstract}
SMARCA4-deficient thoracic sarcoma is a newly described entity of thoracic sarcomas with a poor prognosis, defined by poorly differentiated epithelioid to rhabdoid histomorphology and SMARCA4 gene inactivation. We present a case of a SMARCA4-deficient thoracic sarcoma in a 41-year-old male with a smoking history who presented with an upper anterior mediastinal mass, after seeking medical evaluation for increasing thoracic pain, odynophagia, and dizziness. The biopsy confirmed a large cell tumor with an epithelioid to rhabdoid histomorphology, positive for EMA, CD99, vimentin, TLE1, INI1, PAS-positive cytoplasmic granules, and PD-L1 (100\% of tumor cells). High TMB and HRD scores were displayed in the tumor. The histology and immunophenotype of the mass were in line with the diagnosis of SMARCA4-deficient thoracic sarcoma. In the course of his treatment, the patient showcased a partial response to pembrolizumab and the combination of pembrolizumab and ipilimumab. This case report highlights the importance of recognizing SMARCA4-deficient thoracic sarcoma as an individual entity and supports the importance of checkpoint inhibition therapy for SMARCA4deficient thoracic sarcomas, particularly in cases with a high TMB and PD-L1 expression.
\end{abstract}


Anžič et al.: Ipilimumab and Pembrolizumab Mixed Response in SMARCA4-Deficient Thoracic Sarcoma

\section{Introduction}

SMARCA4 (Switch/sucrose-nonfermenting related [SWI/SNF], matrix associated, actin dependent regulator of chromatin, subfamily a, member 4)-deficient thoracic sarcoma, an aggressive and rare malignancy that presents with rapidly progressive masses involving the lung, mediastinum, and pleura, is a newly proposed histomorphological and molecular entity that was initially described by Sauter et al. [1]. It predominantly affects males with a median age of 48 with a history of smoking. The prognosis is poor, with a median survival of 6-7 months; direct comparison with prognostic data of thymic carcinoma patients reveals a considerably shorter 2-year survival rate (12.5 vs. 64.4\%) [1-5]. Since then several case studies reporting this entity have been described in the literature [1, 6-9].

Histologically, the tumors consist of poorly differentiated epithelioid to rhabdoid cells with high mitotic rates, and present an immunohistochemical loss of the proteins BRG1 (Brahma-related gene-1; the protein encoded by the SMARCA4 gene) and BRM (Brahma), with an intact expression of INI-1 (integrase interactor 1) [2, 4, 10].

BRG1, BRM, and INI-1 are subunits of the SWI/SNF chromatin remodeling complex. SWI/ SNF plays an important role in transcription, replication, DNA repair and recombination, regulation of gene expression, and cell cycle regulation [10]. Due to its direct influence on chromatin accessibility and remodeling, both inactivation as well as overexpression of SWI/ SNF subunits may have carcinogenic potential [10]. BRG1 and BRM are encoded by the genes SMARCA4 and SMARCA2 [4]. Inactivation of SMARCA4 as well as SMARCA2 co-deficiency are characteristic for the tumor profile of SMARCA4-deficient thoracic sarcoma [4]. Generally, mutations in SWI/SNF subunits or associated proteins have been found to be present in approximately $20 \%$ of all human cancers, among them non-small cell lung cancer (NSCLC), medulloblastoma, Burkitt's lymphoma, small cell ovarian carcinoma, hypercalcemic type (SCCOHT), dedifferentiated endometrial carcinoma, sinonasal undifferentiated carcinoma, pediatric rhabdoid tumors, and other cancers [4, 10,11]. SMARCA4 mutations specifically have also been found in cases of thymic carcinoma, NSCLC, and SCCOHT [3, 12-14].

\section{Interdisciplinary Case Presentation}

Initial Presentation and Diagnostics

A 41-year-old man was admitted to our hospital in June 2018 with increasingly worsening thoracic pain, odynophagia, and dizziness that had been lasting for a week. Lab results showed elevated lactate dehydrogenase ( $408 \mathrm{U} / \mathrm{L})$ and increased CRP $(19.7 \mathrm{mg} / \mathrm{L})$. Computerized tomography (CT) of the thorax and abdomen and a consecutive positron emission tomography/CT-fusion (PET/CT) revealed a mass of conglomerated lymph nodes, measuring $5.7 \times 12 \times 10 \mathrm{~cm}$ in the upper anterior mediastinum encompassing infracarinal and hilar lymph nodes, immuring supra-aortic vessels leading to compression of the innominate vein and superior vena cava (Fig. 1).

Subsequently performed lymph node biopsy displayed a monomorphic large cell tumor with an epitheloid to rhabdoid morphology (Fig. 2). Immunohistochemical analyses excluded germ-cell tumor, lymphoma, melanoma, mesothelioma, histiocytic sarcoma, myeloid sarcoma, angiosarcoma, rhabdoid tumor, epithelioid sarcoma, nitrogen regulatory protein 1 (NUT)positive carcinoma, anaplastic lymphoma kinase (ALK)-rearranged malignoma, rhabdomyosarcoma, and mast cell sarcoma. The tumor showed positivity for epithelial membrane antigen (EMA), CD99, vimentin, transducing-like enhancer of split 1 (TLE1), and INI1, and periodic acid Schiff (PAS)-positive cytoplasmic granules. Programmed death-ligand 1 (PD-L1) staining revealed positivity in $100 \%$ of tumor cells (Fig. 2). The tumor presented with considerably high tumor mutation burden (TMB) (674 single nucleotide variants [SNV] and 47 indels) and homologous recombination deficiency (HRD) scores (loss of heterozygosity [LOH]

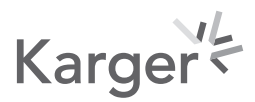




\section{Case Reports in Oncology}

Case Rep Oncol 2021;14:706-715

DOI: $10.1159 / 000515416$

(c) 2021 The Author(s). Published by S. Karger AG, Basel www.karger.com/cro

Anžič et al.: Ipilimumab and Pembrolizumab Mixed Response in SMARCA4-Deficient Thoracic Sarcoma

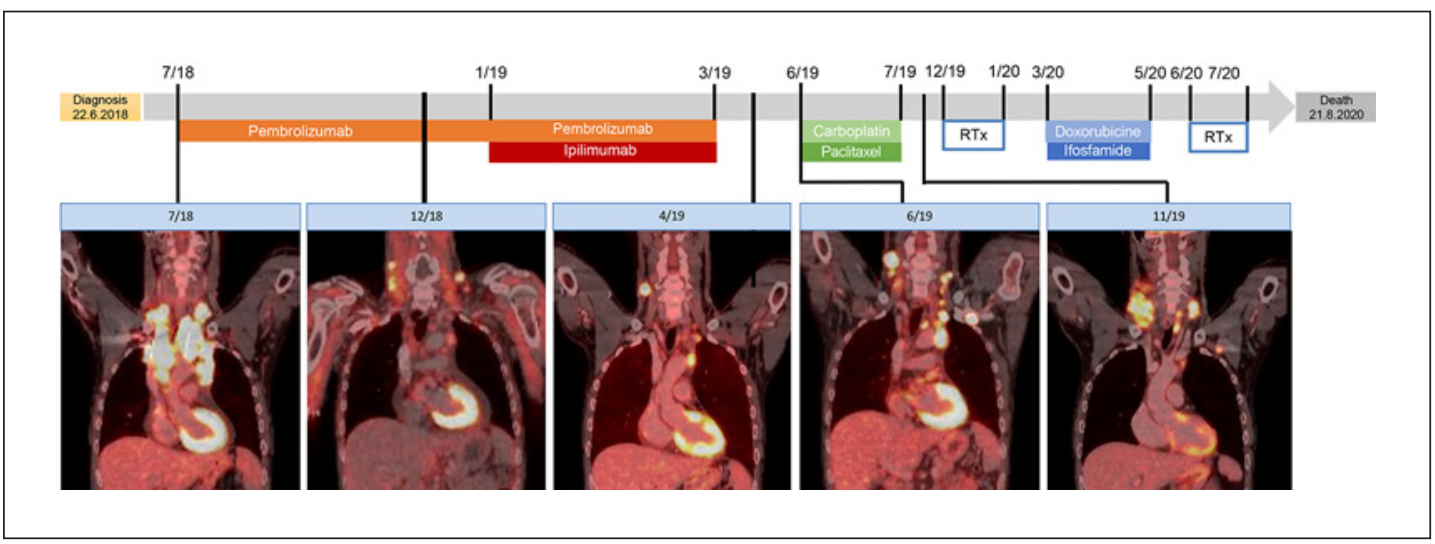

Fig. 1. Treatment timeline and therapeutic response PET-CT follow-ups in a patient with SMARCA4-deficient thoracic sarcoma. Pembrolizumab monotherapy as well as pembrolizumab and ipilimumab combination generated a mixed response (see PET-CT 12/18 and 4/19, respectively). Follow-up from June 2019 onwards showed signs of progressive disease in mediastinal and cervical lymph nodes despite receipt of chemotherapy and radiotherapy. See main text for individual regimens.
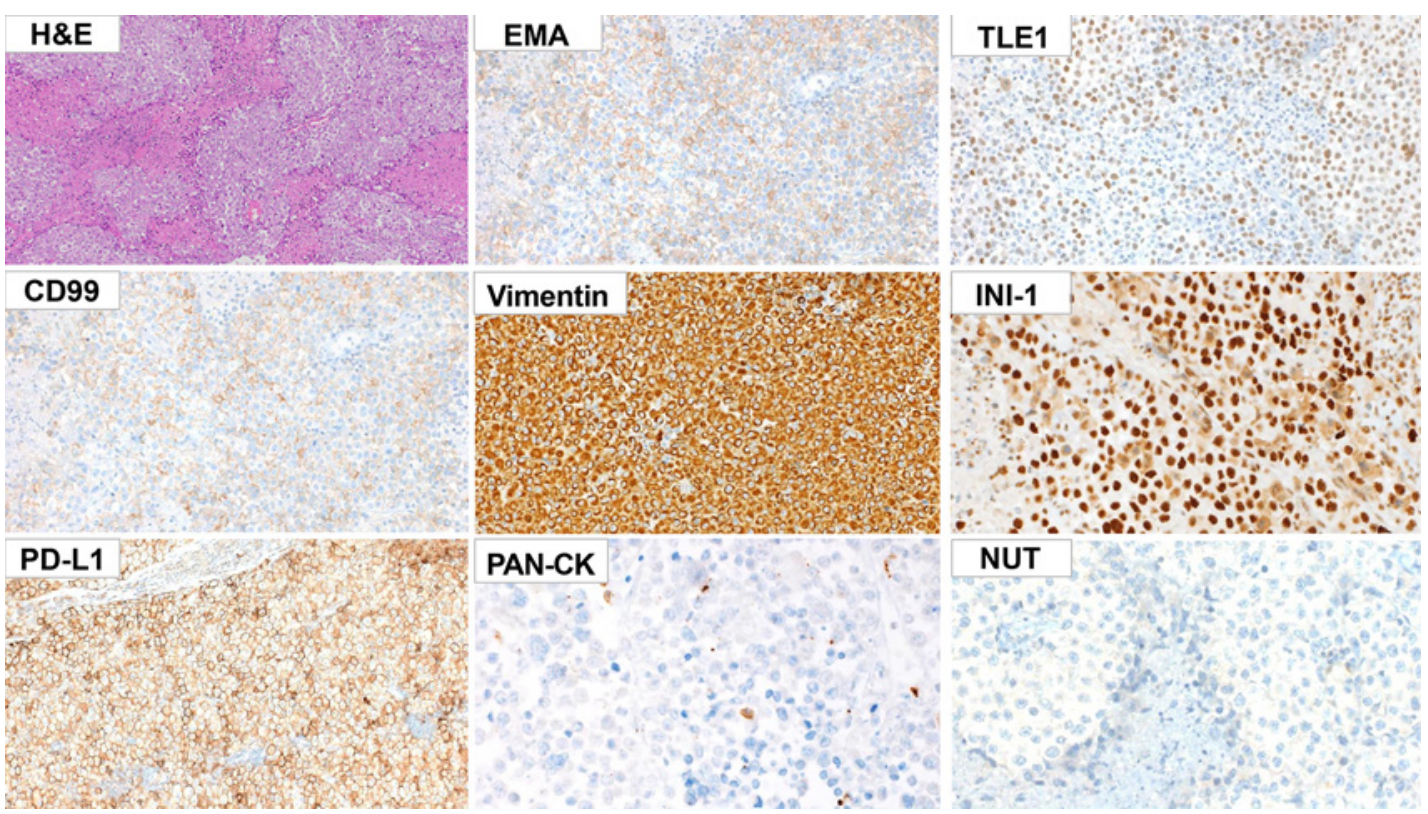

Fig. 2. Microscopic findings of SMARCA4-deficient thoracic sarcoma. Monomorphic, poorly differentiated, large cell tumor with an epithelioid to rhabdoid morphology, strongly positive for EMA, TLE1, CD99, vimentin, INI-1, and focally positive for PAN-CK. PD-L1 was found to be expressed in 100\% of tumor cells. The stain for (I) NUT was negative, excluding NUT carcinoma. Magnification, $\times 100$ for H\&E, EMA, TLE1, CD99, PD-L1, and vimentin; magnification, $\times 200$ for INI-1, PAN-CK, and NUT.

of 20, telomeric-allelic imbalance [TAI] of 22, and large-scale state transitions [LST] of 11). Subsequently performed massive parallel sequencing utilizing the Oncomine ${ }^{\mathrm{TM}}$ Comprehensive Panel Version 3 revealed pathogenic terminating (deleterious) frameshift mutations in Cyclin Dependent Kinase Inhibitor 2A (CDKN2A) (p.T18fs) and SMARCA4 (p.K1334fs). 


\section{Case Reports in Oncology}

Case Rep Oncol 2021;14:706-715

\begin{tabular}{l|l}
\hline DOI: $10.1159 / 000515416$ & @ 2021 The Author(s). Published by S. Karger AG, Basel
\end{tabular} www.karger.com/cro

Anžič et al.: Ipilimumab and Pembrolizumab Mixed Response in SMARCA4-Deficient Thoracic Sarcoma

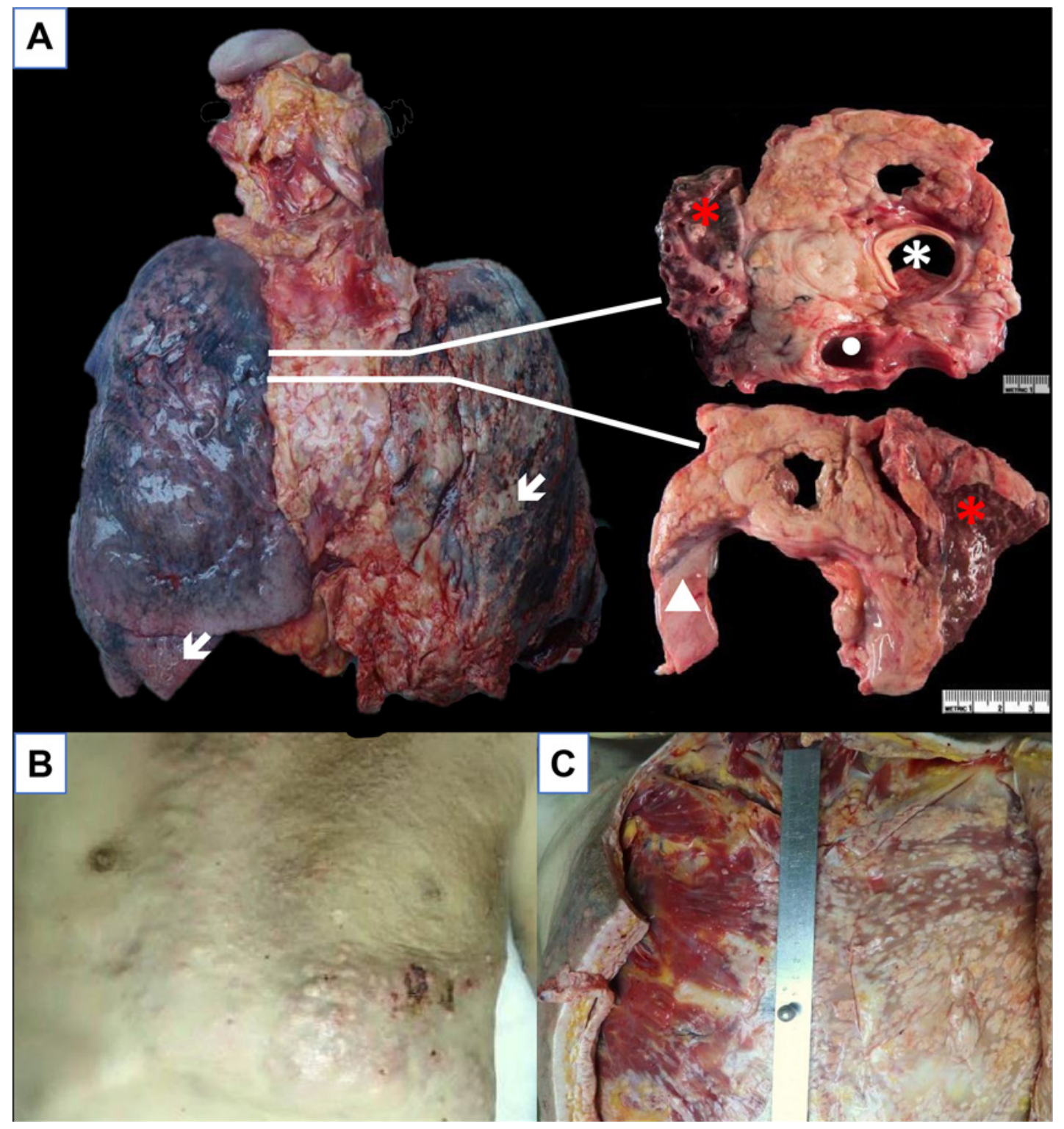

Fig. 3. Gross findings of SMARCA4-deficient thoracic sarcoma at autopsy. A Primary tumor mass $(20 \times 10 \times$ $7 \mathrm{~cm}$ ) in the anterior mediastinum, with diffuse pericardial infiltration (white triangle), per-continuitatem immurement of the left bronchus (white circle) and pulmonary trunk (white asterisk), and diffuse infiltration into the lung parenchyma (red asterisk). Bilateral pleural metastases (white arrows), left > right. B, C Widespread miliar cutaneous and subcutaneous metastases encompassing pectoral muscles of the chest.

Therapeutic Measures and Follow-Up

Figure 1 provides an overview of the therapeutic timeline. In line with the positive PD-L1 status, the patient received 8 cycles of pembrolizumab $200 \mathrm{mg}$ (absolute) and showed a mixed response, with disease progression in cervical lymph nodes and regression in mediastinal lymph nodes. He proceeded to receive 4 cycles of pembrolizumab $200 \mathrm{mg}$ (absolute) and ipilimumab $1 \mathrm{mg} / \mathrm{kg}$ of body weight, which similarly showed a mixed response with progression of the supraclavicular lymph node metastasis and persisting disease in the mediastinal lymph nodes. Three months after receipt of checkpoint inhibitor therapy, palliative 
chemotherapy with 2 cycles of carboplatin AUC and 6 paclitaxel $175 \mathrm{mg} / \mathrm{m}^{2}$, q3 was initiated. Five months later, the patient received palliative radiotherapy for painful lymph node metastases of the cervical and paraclavicular region bilaterally with $18 \times 2.66 \mathrm{~Gy}$. Due to a significant increase in lymph node swelling (tumor progression vs. pseudo-progression), the treatment volume was expanded after 4 fractions. This was followed by palliative chemotherapy with 6 cycles of doxorubicin $75 \mathrm{mg} / \mathrm{m}^{2}$, d1, ifosfamide 1,500 mg/m², d1-5, and G-CSF d7, q3 and a second series of palliative radiotherapy (18 $\times 2.66 \mathrm{~Gy})$ for pain relief. Following that, the treatment volume was expanded after 6 fractions due to increased swelling.

In August 2020, the patient presented with progressive dyspnea, flank pain, and edema of the left arm and ankles. Bronchoalveolar lavage (BAL) excluded an infectious etiology. The patient subsequently developed progressively worsening renal and respiratory failure and died in August 2020.

\section{Autopsy Findings}

An autopsy was performed, revealing a partly necrotic white mass, measuring $20 \times 10 \times$ $7 \mathrm{~cm}$ in the anterior mediastinum, with diffuse pericardial infiltration and per-continuitatem immurement of the left bronchus and pulmonary trunk (Fig. 3a). Metastases were found in cervical, hilar, aortic arch, infrarenal para-aortic, and right retroperitoneal lymph nodes. Pleural carcinomatosis and malignant pleural effusion, in lieu with lymphangiosis carcinomatosa of the pulmonary parenchyma with a few interstitial metastases were present bilaterally. Subcutaneous tubercular metastases of the skin were present on the left side of the neck, chest $(55 \times 30 \mathrm{~cm})$, and left arm with accompanying lymphedema and diffuse infestation of the left pectoral muscle (Fig. 3b-c). Distant metastases included infiltration of the right renal hilus, both adrenal glands, the greater omentum, and jejunal mesothelium.

\section{Discussion}

The patient was a 41-year-old man with a history of smoking and a survival time of 2 years after initial diagnosis, in compliance with the typical epidemiology of SMARCA4-deficient thoracic sarcoma [1,4]. Along with the SMARCA4 mutation, the rhabdoid morphology as well as immunohistochemical profile of the described tumor corresponded to observations from previously conducted studies, histomorphologically and molecularly setting this subgroup apart from non-sarcomatoid SMARCA4-deficient NSCLC. Previously conducted immunohistochemical profiles of thoracic sarcomas demonstrate similarities with our case, particularly in the absence of BRG1 and NUT and positivity for EMA, vimentin, and CD99 $[9,15]$.

The diagnosis of thoracic sarcomas often poses a challenge, typically warranting high expertise. Recent years have witnessed an increased utilization of next-generation sequencing assays as a potential tool for sub-entity recognition, diagnosis, and classification. Mediastinal sarcomas are a thus a notable illustration of interdisciplinary diagnostics by means of genomics and morphology. Current data on treatment strategies for this new and challenging tumor entity is fragmented $[16,17]$. Although surgical resection has reportedly shown survival benefit, a considerable fraction of cases initially presents with locally advanced or metastatic disease. The roles of radiotherapy and adjuvant therapy are still unclear $[18,19]$. Importantly, our patient had showcased a mixed response to treatment with pembrolizumab monotherapy and similarly in combination with ipilimumab that deserves discussion. Previously conducted clinical trials and case studies have shown promise for programmed cell death protein 1 (PD-1) antibody treatment in thymic carcinoma [20]. Two studies on respective patients showed improvement after pembrolizumab treatment in instances with

\section{Karger'}


Anžič et al.: Ipilimumab and Pembrolizumab Mixed Response in SMARCA4-Deficient Thoracic Sarcoma

a high $(>50 \%)$ PD-L1 expression $[21,22]$. Although data on sarcomatoid mediastinal tumors is scarce, numerous trials have failed to prove consistent clinical efficacy of immune checkpoint inhibitors (ICI) [23]. In two cases of SMARCA4-deficient thoracic tumors, there have been isolated reports of a marked treatment response to pembrolizumab despite heterogeneity of PD-L1 expression: in one of the cases, the tumor displayed immunohistochemical negativity for PD-L1, while in the other, $60 \%$ of tumor cells expressed PD-L1 [24, 25]. This discrepancy between PD-L1 expression and treatment response implies a more complex situation respecting prediction of ICI efficacy in SMARCA4-deficient tumors, which warrants further investigation [26].

There is considerable clinical evidence of responses to anti-PD-1 agents in a broad spectrum of other SMARCA4-deficient tumor entities. In four cases of SMARCA4-deficient SCCOHT, prolonged responses to nivolumab and pembrolizumab have been reported [27]. In a case of SMARCA4-deficient lung adenocarcinoma, negative for PD-L1, the patient received nivolumab and showcased a marked reduction of the lung metastases for 14 months [28]. In vitro evidence has suggested plausible pathophysiological rationale regarding this phenomenon. Mutations in the polybromo-associated BAF (PBAF) form of the SWI/SNF complex were reported to increase tumor cell line sensitivity to interferon- $\gamma$, resulting in enhanced secretion of chemokines essential for effector T-cell chemotaxis [29]. Another study reported an interaction between the SWI/SNF complex gene AT-rich interaction domain 1A (ARID1A) and MutS homolog 2 (MSH2), implying aberrant mismatch repair and generally increased genomic instability that may generate a higher TMB, which potentiates a more effective immunotherapy response [30]. Our patient, too, had an extremely high TMB; moreover, in several studies where patients had received ICI therapy, a higher survival benefit has been observed in patients with a higher TMB, compared to those with a lower TMB [31, 32]. This was also demonstrated in cases of patients treated with a combination of two ICIs, again, independently of PD-L1 expression [33, 34]. The use of combined ICIs in various cancers may result in an improvement of overall survival [35]. Cytotoxic T-lymphocyte-associated protein 4 (CTLA4) is associated with T-cell proliferation regulation in the early stages of an immune response occurring in lymph nodes, while PD- 1 is thought to play part in later stages occurring in the tumor microenvironment [36]. Amplified disease response to dual ICI therapy compared to single ICI therapy has been observed in advanced melanomas, metastatic renal cell cancers (RCC), small-cell lung cancers (SCLC), and NSCLC $[37,38]$.

Recent in vitro studies have described synthetic lethality of ICI with cyclin-dependent kinase 4/6 (CDK4/6) inhibition associated with a downregulation of cyclin D1 in SMARCA4deficient NSCLC, thus additionally introducing CDK4/6 inhibitors such as palbociclib or abemaciclib as potential treatment options in SMARCA4-deficient tumors [39, 40]. Although no randomized controlled trials have been performed yet, a case study reported promising response of combination treatment with nivolumab and abemaciclib in SCCOHT. SMARCA4-deficiency leads to reduced CDK4/6 kinase activity, which in turn leads to cytostasis and increased antigen presentation in tumor cells, whilst decreasing expression of inhibitory receptors and promoting chemokine secretion in immune cells, which propagates T-cell infiltration and their function as effectors $[27,41]$. Combination of ICI and CDK4/ 6 inhibitor therapy may thus possibly be an effective treatment alternative in SMARCA4-deficient malignancies, and may thus hold promise for the treatment of SMARCA4-deficient thoracic sarcoma [27]. CDK4/6 inhibitors, furthermore, could demonstrate potential therapeutic efficacy in cancers with a mutation in $C D K N 2 A$, analogously present in our patient, since $C D K N 2 A$ is a negative regulator of CDK4/6. Different cancers with $C D K N 2 A$ loss have been found to show an intermediate level of sensitivity to CDK4/6 inhibitors, displaying growth cessation, but not tumor regression [42-46]. In our

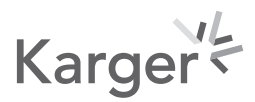


case, a CDK4/6 inhibitor therapy had been planned but was unable to be initiated as the patient had deteriorated too quickly.

Another promising treatment approach could also encompass a combination of poly (ADP-ribose) polymerase (PARP) inhibitors and ICI, which has shown promise in a study of patients with advanced solid tumors [47]. PARP inhibition leads to dysregulated DNA repair due to aberrant homologous recombination [48]. It furthermore restores an efficient Th1 immune response and promotes PD-L1 expression, which could benefit and increase the effectiveness of ICIs $[49,50]$. PARP inhibitors monotherapy has also shown promise in the treatment of different cancers with high HRD scores [51-53].

New studies have introduced an inhibition of enhancer of zeste homolog 2 (EZH2), a histone methyltransferase, as a further emerging treatment possibility for tumors with defects in the SWI/SNF complex. EZH2 is a catalytic subunit of the polycomb repressor complex (PRC) that is involved in cancer proliferation and metastasis, while SWI/SNF regulates the action of PRC. Targeted EZH2 inhibition may thus downregulate the PRC complex and halt cancer growth. Currently, a clinical trial investigating the efficacy of EZH2 inhibitors in patients with SMARCA4-deficient thoracic sarcomas is underway [1,54].

In conclusion, this is one of the few case reports delineating partial response to pembrolizumab/ipilimumab combination therapy in SMARCA4-deficient thoracic sarcoma. Our findings underline the importance of recognizing this subset of tumors as an individual disease entity, and support the inherent value of checkpoint inhibitor therapy particularly in cases with high PD-L1 expression and TMB. Further studies are warranted to characterize the histomorphological and genomic landscape of these aggressive thoracic tumors to optimize novel therapeutic approaches.

\section{Acknowledgements}

The authors would like to thank the patient and his relatives, as well as Martin Portmann for the photographs of gross findings, Oscar Herrera for assistance at autopsy, and Luigi Terraciano, Thomas Menter, Nick Deigendesch, and Jürgen Hench for contributing their diagnostic advice.

\section{Statement of Ethics}

Written informed consent for publication of this case report and any accompanying images was obtained from the patient before his demise.

\section{Conflict of Interest Statement}

The authors have no conflicts of interest to declare.

\section{Funding Sources}

Nothing to declare. 


\section{Case Reports in Oncology}

\begin{tabular}{l|l}
\hline Case Rep Oncol 2021;14:706-715 \\
\hline DOI: 10.1159/000515416 & $\begin{array}{l}\text { ○ 2021 The Author(s). Published by S. Karger AG, Basel } \\
\text { www.karger.com/cro }\end{array}$ \\
\hline
\end{tabular}

Anžič et al.: Ipilimumab and Pembrolizumab Mixed Response in SMARCA4-Deficient Thoracic Sarcoma

\section{Author Contributions}

J.D.H. and A.T. conceived the work and provided the histological diagnosis and analysis for the patient. J.D.H. performed the autopsy. Manuscript by N.A. and J.D.H. N.A. collected clinical patient data and designed images. F.K. and A.-L.E. provided details of therapy regimens and clinical disease progression/management. Critical revision for intellectual content by J.D.H. and A.T. All authors read and approved the final manuscript.

\section{References}

1 Sauter JL, Graham RP, Larsen BT, Jenkins SM, Roden AC, Boland JM. SMARCA4-deficient thoracic sarcoma: a distinctive clinicopathological entity with undifferentiated rhabdoid morphology and aggressive behavior. Mod Pathol. 2017;30(10):1422-32.

2 Takeda M, Tani Y, Saijo N, Shimizu S, Taniguchi Y, Otsuka K, et al. Cytopathological Features of SMARCA4Deficient Thoracic Sarcoma: Report of 2 Cases and Review of the Literature. Int J Surg Pathol. 2020;28(1): 109-14.

3 Le Loarer F, Watson S, Pierron G, de Montpreville VT, Ballet S, Firmin N, et al. SMARCA4 inactivation defines a group of undifferentiated thoracic malignancies transcriptionally related to BAF-deficient sarcomas. Nat Genet. 2015;47(10):1200-5.

4 Stewart BD, Kaye F, Machuca T, Mehta HJ, Mohammed T-L, Newsom KJ, et al. SMARCA4-Deficient Thoracic Sarcoma: A Case Report and Review of Literature. Int J Surg Pathol. 2020;28(1):102-8.

5 Perret R, Chalabreysse L, Watson S, Serre I, Garcia S, Forest F, et al. SMARCA4-deficient Thoracic Sarcomas: Clinicopathologic Study of 30 Cases With an Emphasis on Their Nosology and Differential Diagnoses. Am J Surg Pathol.2019;43(4):455-65.[not available]

6 Nambirajan A, Dutta R, Malik PS, Bubendorf L, Jain D. Cytology of SMARCA4-Deficient Thoracic Neoplasms: Comparative Analysis of SMARCA4-Deficient Non-Small Cell Lung Carcinomas and SMARCA4-Deficient Thoracic Sarcomas. Acta Cytol. 2021;65(1):67-74.

7 Iijima Y, Sakakibara R, Ishizuka M, Honda T, Shirai T, Okamoto T, et al. Notable response to nivolumab during the treatment of SMARCA4-deficient thoracic sarcoma: a case report. Immunotherapy. 2020;12(8):563-9.

8 Decroix E, Leroy K, Wislez M, Fournel L, Alifano M, Damotte D, et al. SMARCA4-deficient thoracic tumors: A new entity. Bull Cancer.2020;107(1):41-7.[not available]

9 Rekhtman N, Montecalvo J, Chang JC, Alex D, Ptashkin RN, Ai N, et al. SMARCA4-Deficient Thoracic Sarcomatoid Tumors Represent Primarily Smoking-Related Undifferentiated Carcinomas Rather Than Primary Thoracic Sarcomas. J Thorac Oncol. 2020;15(2):231-47.

10 Wu Q, Lian JB, Stein JL, Stein GS, Nickerson JA, Imbalzano AN. The BRG1 ATPase of human SWI/SNF chromatin remodeling enzymes as a driver of cancer. Epigenomics. 2017;9(6):919-31.

11 Versteege I, Sévenet N, Lange J, Rousseau-Merck MF, Ambros P, Handgretinger R, et al. Truncating mutations of hSNF5/INI1 in aggressive paediatric cancer. Nature. 1998 9;394(6689):203-6.

12 Wang Y, Thomas A, Lau C, Rajan A, Zhu Y, Killian JK, et al. Mutations of epigenetic regulatory genes are common in thymic carcinomas. Sci Rep. 2014;4:7336.

13 Herpel E, Rieker RJ, Dienemann H, Muley T, Meister M, Hartmann A, et al. SMARCA4 and SMARCA2 deficiency in non-small cell lung cancer: immunohistochemical survey of 316 consecutive specimens. Ann Diagn Pathol. 2017;26:47-51.

14 Schoenfeld AJ, Bandlamudi C, Lavery JA, Montecalvo J, Namakydoust A, Rizvi H, et al. The Genomic Landscape of SMARCA4 Alterations and Associations with Outcomes in Patients with Lung Cancer. Clin Cancer Res. 2020; 26(21):5701-8.

15 Nambirajan A, Dutta R, Malik PS, Bubendorf L, Jain D. Cytology of SMARCA4-Deficient Thoracic Neoplasms: Comparative Analysis of SMARCA4-Deficient Non-Small Cell Lung Carcinomas and SMARCA4-Deficient Thoracic Sarcomas. Acta Cytol. 2020:1-8.[not available in pubmed,crossref]

16 Skubitz KM, Pambuccian S, Manivel JC, Skubitz AP. Identification of heterogeneity among soft tissue sarcomas by gene expression profiles from different tumors. J Transl Med. 2008;6:23.

17 Palmieri G, Tortora M, Parola S, Picozzi F, Ottaviano M. Mediastinal soft tissue sarcoma: dark sides and future lights. Mediastinum. June. 2020;4:9.[not available in pubmed,crossref]

18 Paquette M, Truong PT, Hart J, Jones SO, Martens B, Christie JL, et al. Primary sarcoma of the mediastinum: a report of 16 cases referred to the British Columbia Cancer Agency. J Thorac Oncol. 2010 Jun;5(6):898-906.

19 Engelhardt KE, DeCamp MM, Yang AD, Bilimoria KY, Odell DD. Treatment Approaches and Outcomes for Primary Mediastinal Sarcoma: Analysis of 976 Patients. Ann Thorac Surg. 2018;106(2):333-9.

20 Isshiki T, Isobe K, Tochigi N, Sunakawa M, Nakamura Y, Shibuya K, et al. Successful Use of Pembrolizumab to Treat Refractory Thymic Carcinoma with High PD-L1 Expression. Case Rep Oncol. 2018 Sep-Dec;11(3):68892. 
21 Giaccone G, Kim C, Thompson J, McGuire C, Kallakury B, Chahine JJ, et al. Pembrolizumab in patients with thymic carcinoma: a single-arm, single-centre, phase 2 study. Lancet Oncol. 2018;19(3):347-55.

22 Cho J, Kim HS, Ku BM, Choi YL, Cristescu R, Han J, et al. Pembrolizumab for Patients With Refractory or Relapsed Thymic Epithelial Tumor: An Open-Label Phase II Trial. J Clin Oncol. 2019 Aug 20;37(24):2162-70.

23 Toulmonde M, Penel N, Adam J, Chevreau C, Blay JY, Le Cesne A, et al. Use of PD-1 Targeting, Macrophage Infiltration, and IDO Pathway Activation in Sarcomas: A Phase 2 Clinical Trial. JAMA Oncol. 2018;4(1):93-7.

24 Takada K, Sugita S, Murase K, Kikuchi T, Oomori G, Ito R, et al. Exceptionally rapid response to pembrolizumab in a SMARCA4-deficient thoracic sarcoma overexpressing PD-L1: A case report. Thorac Cancer. 2019;10(12): 2312-5.

25 Henon C, Blay JY, Massard C, Mir O, Bahleda R, Dumont S, et al. Long lasting major response to pembrolizumab in a thoracic malignant rhabdoid-like SMARCA4-deficient tumor. Ann Oncol. 2019;30(8):1401-3.

26 Jelinic P, Ricca J, Van Oudenhove E, Olvera N, Merghoub T, Levine DA, et al. Immune-Active Microenvironment in Small Cell Carcinoma of the Ovary, Hypercalcemic Type: Rationale for Immune Checkpoint Blockade. J Natl Cancer Inst. 2018;110(7):787-90.

27 Lee EK, Esselen KM, Kolin DL, Lee LJ, Matulonis UA, Konstantinopoulos PA. Combined CDK4/6 and PD-1 Inhibition in Refractory SMARCA4-Deficient Small-Cell Carcinoma of the Ovary, Hypercalcemic Type. JCO Precis Oncol. 2020;4:736-42.

28 Naito T, Umemura S, Nakamura H, Zenke Y, Udagawa H, Kirita K, et al. Successful treatment with nivolumab for SMARCA4-deficient non-small cell lung carcinoma with a high tumor mutation burden: A case report. Thorac Cancer. 2019;10(5):1285-8.

29 Pan D, Kobayashi A, Jiang P, Ferrari de Andrade L, Tay RE, Luoma AM, et al. A major chromatin regulator determines resistance of tumor cells to T cell-mediated killing. Science. 2018;359(6377):770-5.

30 Shen J, Ju Z, Zhao W, Wang L, Peng Y, Ge Z, et al. ARID1A deficiency promotes mutability and potentiates therapeutic antitumor immunity unleashed by immune checkpoint blockade. Nat Med. 2018 May;24(5):556-62.

31 Rizvi NA, Hellmann MD, Snyder A, Kvistborg P, Makarov V, Havel JJ, et al. Cancer immunology. Mutational landscape determines sensitivity to PD-1 blockade in non-small cell lung cancer. Science. 2015;348(6230):124-8.

32 Samstein RA-O, Lee CH, Shoushtari AA-O, Hellmann MA-O, Shen R, Janjigian YY, et al. Tumor mutational load predicts survival after immunotherapy across multiple cancer types. Nat Genet. 2019(51):202-6.[not available in pubmed,crossref]

33 Ready N, Hellmann MD, Awad MM, Otterson GA, Gutierrez M, Gainor JF, et al. First-Line Nivolumab Plus Ipilimumab in Advanced Non-Small-Cell Lung Cancer (CheckMate 568): Outcomes by Programmed Death Ligand 1 and Tumor Mutational Burden as Biomarkers. J Clin Oncol. 2019 Apr 20;37(12):992-1000.

34 Reck M, Schenker M, Lee KH, Provencio M, Nishio M, Lesniewski-Kmak K, et al. Nivolumab plus ipilimumab versus chemotherapy as first-line treatment in advanced non-small-cell lung cancer with high tumour mutational burden: patient-reported outcomes results from the randomised, open-label, phase III CheckMate 227 trial. Eur J Cancer. 2019 Jul;116:137-47.

35 Chae YK, Arya A, Iams W, Cruz MR, Chandra S, Choi J, et al. Current landscape and future of dual anti-CTLA4 and PD-1/PD-L1 blockade immunotherapy in cancer; lessons learned from clinical trials with melanoma and non-small cell lung cancer (NSCLC). J Immunother Cancer. 2018;6(1):39.

36 Buchbinder EI, Desai A. CTLA-4 and PD-1 Pathways: Similarities, Differences, and Implications of Their Inhibition. Am J Clin Oncol. 2016;39(1):98-106.

37 Lim SM, Hong MH, Kim HR. Immunotherapy for Non-small Cell Lung Cancer: Current Landscape and Future Perspectives. Immune Netw. 2020;20(1):e10-e.

38 Hellmann MD, Ciuleanu TE, Pluzanski A, Lee JS, Otterson GA, Audigier-Valette C, et al. Nivolumab plus Ipilimumab in Lung Cancer with a High Tumor Mutational Burden. N Engl J Med. 2018;378(22):2093-104.

39 Xue Y, Meehan B, Macdonald E, Venneti S, Wang XQD, Witkowski L, et al. CDK4/6 inhibitors target SMARCA4determined cyclin D1 deficiency in hypercalcemic small cell carcinoma of the ovary. Nat Commun. 2019;10(1): 558.

40 Xue Y, Meehan B, Fu Z, Wang XQD, Fiset PO, Rieker R, et al. SMARCA4 loss is synthetic lethal with CDK4/6 inhibition in non-small cell lung cancer. Nat Commun. 2019;10(1):557.

41 Xue Y, Meehan B, Fu Z, Wang XQD, Fiset PO, Rieker R, et al. SMARCA4 loss is synthetic lethal with CDK4/6 inhibition in non-small cell lung cancer. Nat Commun. 2019;10(1):557.

42 Gong X, Litchfield LM, Webster Y, Chio LC, Wong SS, Stewart TR, et al. Genomic Aberrations that Activate D-type Cyclins Are Associated with Enhanced Sensitivity to the CDK4 and CDK6 Inhibitor Abemaciclib. Cancer Cell. 2017;32(6):761-e6.

43 Patnaik A, Rosen LS, Tolaney SM, Tolcher AW, Goldman JW, Gandhi L, et al. Efficacy and Safety of Abemaciclib, an Inhibitor of CDK4 and CDK6, for Patients with Breast Cancer, Non-Small Cell Lung Cancer, and Other Solid Tumors. Cancer Discov. 2016;6(7):740.

44 Wiedemeyer WR, Dunn IF, Quayle SN, Zhang J, Chheda MG, Dunn GP, et al. Pattern of retinoblastoma pathway inactivation dictates response to CDK4/6 inhibition in GBM. Proc Natl Acad Sci USA. 2010;107(25):11501.

45 Konecny GE, Winterhoff B, Kolarova T, Qi J, Manivong K, Dering J, et al. Expression of p16 and retinoblastoma determines response to CDK4/6 inhibition in ovarian cancer. Clin Cancer Res. 2011;17(6):1591-602.

46 Ahn ER, Mangat PK, Garrett-Mayer E, Halabi S, Dib EG, Haggstrom DE, et al. Palbociclib in Patients With NonSmall-Cell Lung Cancer With CDKN2A Alterations: Results From the Targeted Agent and Profiling Utilization Registry Study. JCO Precision Oncology. 2020(4):757-66.[not available] 
47 Friedlander M, Meniawy T, Markman B, Mileshkin LR, Harnett PR, Millward M, et al. A phase 1b study of the anti-PD-1 monoclonal antibody BGB-A317 (A317) in combination with the PARP inhibitor BGB-290 (290) in advanced solid tumors. J Clin Oncol. 2017;35(15_Suppl 1):3013.

48 Ashworth A. A synthetic lethal therapeutic approach: poly(ADP) ribose polymerase inhibitors for the treatment of cancers deficient in DNA double-strand break repair. J Clin Oncol. 2008;26(22):3785-90.

49 Peyraud F, Italiano A. Combined PARP Inhibition and Immune Checkpoint Therapy in Solid Tumors. Cancers. 2020;12(6):1502.

50 Jiao S, Xia W, Yamaguchi H, Wei Y, Chen MK, Hsu JM, et al. PARP Inhibitor Upregulates PD-L1 Expression and Enhances Cancer-Associated Immunosuppression. Clin Cancer Res. 2017;23(14):3711-20.

51 Mirza MR, Monk BJ, Herrstedt J, Oza AM, Mahner S, Redondo A, et al. Niraparib Maintenance Therapy in Platinum-Sensitive, Recurrent Ovarian Cancer. N Engl J Med. 2016 Dec 1;375(22):2154-64.

52 Coleman RL, Oza AM, Lorusso D, Aghajanian C, Oaknin A, Dean A, et al. Rucaparib maintenance treatment for recurrent ovarian carcinoma after response to platinum therapy (ARIEL3): a randomised, double-blind, placebo-controlled, phase 3 trial. Lancet. 2017 Oct 28;390(10106):1949-61.

53 Pujade-Lauraine E, Ledermann JA, Selle F, Gebski V, Penson RT, Oza AM, et al. Olaparib tablets as maintenance therapy in patients with platinum-sensitive, relapsed ovarian cancer and a BRCA1/2 mutation (SOLO2/ ENGOT-Ov21): a double-blind, randomised, placebo-controlled, phase 3 trial. Lancet Oncol. 2017 Sep;18(9): 1274-84.

54 Wu S, Fatkhutdinov N, Fukumoto T, Bitler BG, Park PH, Kossenkov AV, et al. SWI/SNF catalytic subunits' switch drives resistance to EZH2 inhibitors in ARID1A-mutated cells. Nat Commun. 2018;9(1):4116. 